\title{
Attitude of Burundi Doctors Regarding Physical Activity Prescription in Case of Musculoskeletal Problem Related to Pregnancy
}

\author{
NGAYIMBESHA Adrien*, BIZIMANA Jean Berchmans, GAKIMA Marie Stella and \\ NZISABIRA Felix
}

Institute of Physical Education and Sports, University of Burundi, Burundi

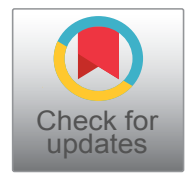

*Corresponding author: NGAYIMBESHA Adrien, Institute of Physical Education and Sports, University of Burundi, Burundi, Tel: +25776437965; +2579924813, E-mail: adrienngayimbesha@yahoo.fr

\begin{abstract}
Introduction: Exercise during pregnancy not only helps reduce backache, fatigue and swelling of the extremities, the use of physical activity during pregnancy also improves women's sleep, mood and posture.

Aim: The present study aimed to know practices, attitudes and knowledge of Burundi doctors a regarding exercise prescription during pregnancy.

Method: Data used in the present study confection have been gathered using a self-report questionnaire developed by us.

Results: $90.4 \%$ of Burundi doctors $(n=95)$ affirmed that they do never prescribe physical exercise nor advice physical exercise to pregnant woman in consultation. All questioned Burundi doctors didn't agree to the importance of physical exercise prescription for the prevention and management of musculoskeletal problems during pregnancy. Results have showed that $92.38 \%(n=97)$ do not know any more the international recommendation on physical exercise practice.
\end{abstract}

Conclusion: Physical exercise prescription is not integrated in everyday primary care particularly in case of pregnancy.

\section{Keywords}

Pregnant women, Doctors, Physical activity prescription, Musculoskeletal problems

\section{Introduction}

In the absence of medical or obstetrical complications, pregnancy should be a period of joy for every woman. However, during this period, many physiological and anatomical changes occur, and which impose a negative impact on woman's health. The gradually changing body and increasing weight of the pregnant woman cause noticeable alterations in her posture and in the way her walks [1].

The abdominal distention during pregnancy gives the pelvis a forward tilt, decreased abdominal muscle tones, and the increased weight require a realignment of a spinal curvature late in pregnancy. As consequence, the pregnant woman's center of gravity shifts forward, and this accentuate the lumbar and dorsal curves. Moreover, ligamentous and muscular structures of the middle and lower spine may be severely stressed [2]. As the pregnancy progresses, the most common foot problems occurring during pregnancy include edema, over-pronation, toe nail changes, leg pain, varicose veins and pain in the arches and heels [3]. These changes related to pregnancy often cause musculoskeletal discomfort. Research result's shows that up to $75 \%$ of pregnant women have been reported to complain of back and foot pain problems [4].

A Cochrane systematic review [5] reports that more than two-thirds of pregnant women experience low back pain during pregnancy $(66 \%)$ and almost $20 \%$ experience pelvic pain. The disability and distress caused by the lower back pain and pelvic pain added to other anatomical changes to foot during pregnancy are increasingly being recognized as an issue that needs to be addressed. This is mainly due to the reporting of research that demonstrates the impact on daily living for women; reports indicate disability at home and in the 
workplace, insomnia and increased risk of developing depression [6] and [7]. These musculoskeletal problems related to pregnancy are accentuated by the sedentary lifestyle.

There is consistent evidence that promoting physical activity in women of reproductive age may be a promising approach to prevent excessive weight gain, gestational diabetes mellitus, and subsequent complications suffered by children born from mothers with gestational diabetes $[8,9]$. Exercise during pregnancy not only helps reduce backache, fatigue and swelling of the extremities, it also improves women's sleep, mood, and posture $[10,11]$. With the ongoing increase of chronic disease and musculoskeletal problems, the World Health Organization (WHO) recommend urgent preventative action and this action must include an increased emphasis on physical activity as a public health and medical intervention [12]. Health care professionals are therefore being called upon to become more aggressive in implementing physical activity recommendations [13]. In some countries, physical activity is now used as a preventative tool for many diseases. For example, Physical activity on prescription has been in use since the eighties in Sweden and New Zealand [14]. The use of the method has grown in recent years and was applied by all county councils in Sweden in 2008 [15]. During the past few years, the method has been introduced in many countries around the world [15].

In USA for example, a survey conducted among primary care practitioner in 2006 reported that $47 \%$ of primary care provider did not prescribe exercise to their patient at the time of the research [16]. This shows that a disparity does exist among health care providers regarding to exercise belief and habit.

While some insight has been gained into the practices and attitudes of some countries doctors towards exercise prescription, there is no information about the exercise prescription practices, knowledge and attitudes in Burundi doctors. In this situation of lack of information many questions are asked:

1. Do Burundi doctors prescribe exercise to their patients pregnant as a part of a standard consultation?

2. What are the attitudes of Burundi doctors towards exercise prescription especially in case of pregnancy?

3. Do Burundi doctors have the basic knowledge needed to compile a safe and effective exercise prescription?

4. The present study aimed to know practices, attitudes and knowledge of Burundi doctors a regarding exercise prescription during pregnancy.

\section{Method}

\section{Study participants}

Targeted population for the present study was doc- tors attached in maternity and gyneco-obstetric department in public hospitals situated in urban; semi-urban area and rural area. A total of 105 doctors currently in service of maternity have been recruited and accepted to participate to the study.

Participant's distribution regarding working area is as follow: 48 doctors work in urban area, 41 doctors in semi-urban area and 16 doctors participating in the present study work in rural area.

\section{Inclusion criteria}

$$
\begin{aligned}
& \checkmark \text { To be qualified as medical doctor } \\
& \checkmark \text { To be attached in maternity service } \\
& \checkmark \text { To work in public hospital } \\
& \checkmark \text { To accept to participate in the study freely }
\end{aligned}
$$

\section{Exclusion criteria}

$\checkmark$ To work in private sector

$\checkmark$ To fail to complete self-report questionnaire

$\checkmark$ To be attached in service other than maternity

$\checkmark$ To don't have medical doctor qualification

\section{Study design}

This study was a descriptive study to assess the knowledge, practices and attitudes of Burundi doctors providing health care in the department of maternity and related health problems regarding exercise prescription as preventive and even therapy in case of musculoskeletal problems inherent to pregnancy. Once the study approved by the Ethics Committee of the Faculty of medicine of Burundi national university, strategy to conduct the study has been developed. The group of participants has been approached and participation acceptation for doctors has been obtained during the week dedicated to health in Burundi held on $25^{\text {th }}$ April 2017. The self-report questionnaire for each doctor was distributed during the same week.

After collecting back completed questionnaire the researcher grouped the data into different assessment areas after which the data were analyzed.

\section{Data collection}

To gather date used in this study has been collected using self-report questionnaire. The used questionnaire was developed by us. A pilot study was organized in way to expose any possible confusion or ambiguity during completion with the ultimately goal of determination of questionnaire validity. Questionnaire amendments were then made according to pilot study comments. The questionnaire was divided into three different parties:

$\checkmark$ Practices of doctors and regarding exercise program

$\checkmark$ Attitude of Burundi doctors toward exercise prescription 


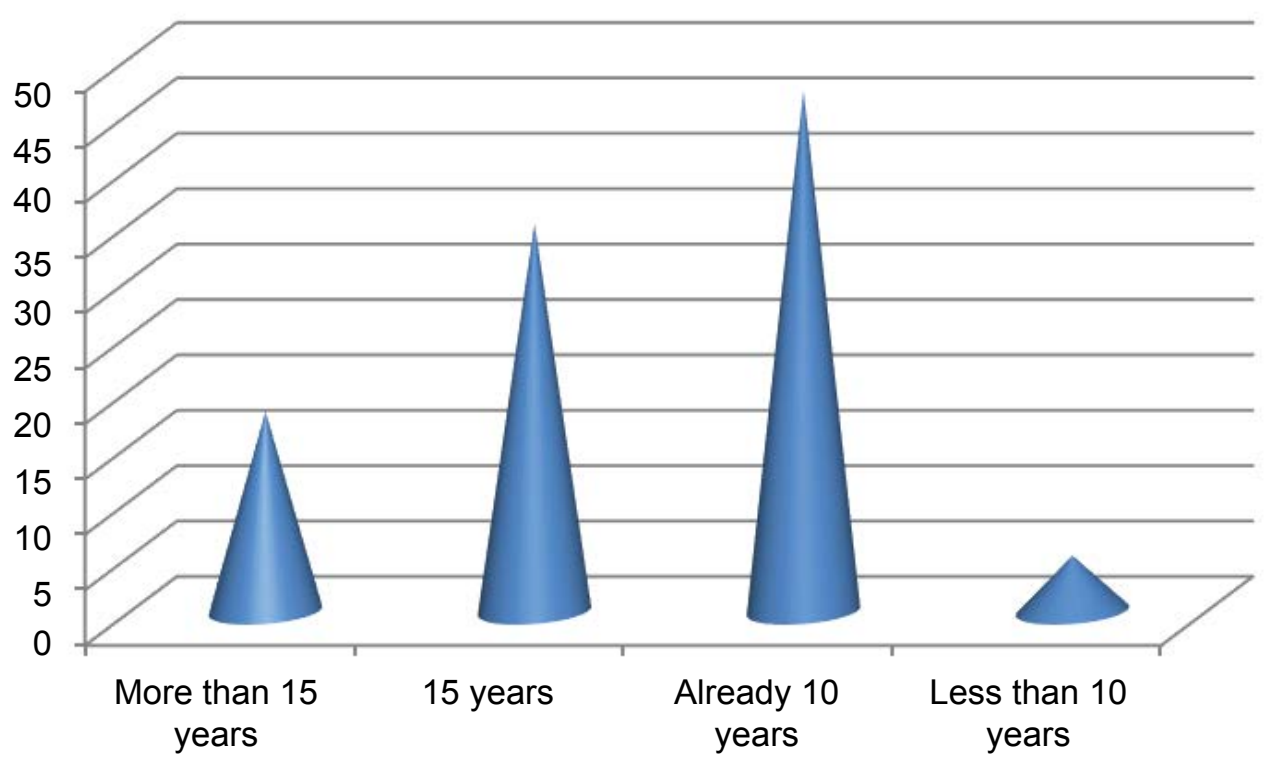

Figure 1: Experiences in practicing medicine.

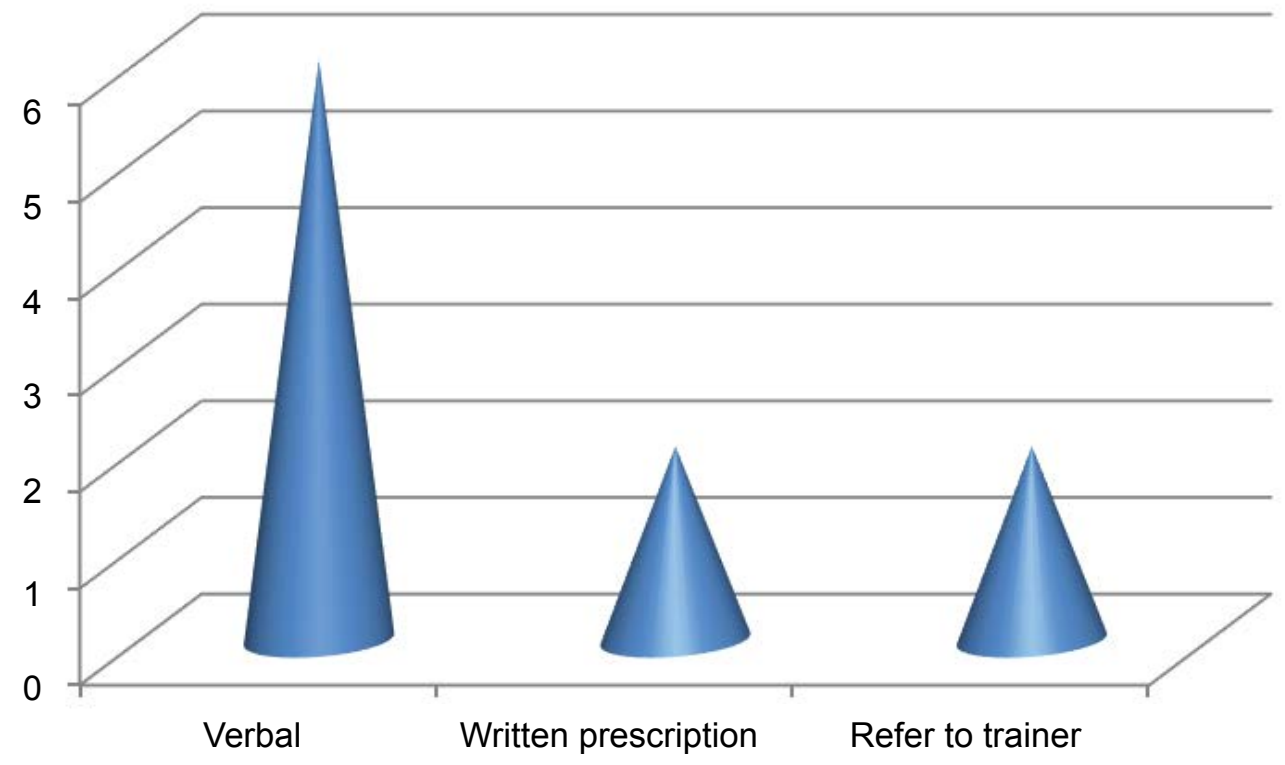

Figure 2: Mode of physical exercise prescription.

Knowledge of international recommendation regarding physical exercise practice

\section{Statistical analysis}

Due to the nature of the study (descriptive study), descriptive statistics was mainly used in data analysis and discussion.

\section{Ethical consideration}

The ethic committee of the faculty of medicine within the Burundi University approved the study method and protocol.

\section{Results}

In way to explore whether years of practicing medicine was a possible influential factor in their knowledge or attitudes towards exercise prescription, the partici- pants were asked to declare the year in which they began their career of health care in department of maternity. Of the doctors that responded to our questionnaire, $17.14 \%(n=18)$ has more than 15 years of practicing medicine; $33.33 \%$ of respondent $(n=35)$ has 15 years of practicing medicine. $44.76 \%$ of doctors participating to the present study $(n=47)$ has begun their career of medicine 10 years ago. $4.7 \%(n=5)$ has an experience of less than 10 years. Figure 1 shows number of year's career in medicine.

For the exercise prescription practice, the big majority of doctors who responded to our questionnaire claimed that their do never prescribe physical activity to pregnant women. In fact, $90.4 \%$ of respondent doctors $(n=95)$ affirmed that they do never prescribe physical exercise nor advice physical exercise to pregnant woman in consultation. A very negligible number of doctors 
to prescribe physical exercise to woman come in consultation. For these doctors, it is important to assess the frequency and modality of prescribing exercise to their patients and the way that prescription is done.

Results have showed that $5.7 \%(n=6)$ of doctors provide a verbal exercise advice as exercise prescription; only $1.9 \%(n=2)$ gave a written prescription of physical exercise to their patients. Another $1.9 \%(n=2)$ refers their patients requiring physical exercise to physical trainers.

The analysis of result showed a very little extend of exercise prescription. In fact, this prescription is approximately done to $1-6 \%$ of pregnant patient. Other prescription may concern nutrition and vaccination side.

Furthermore, study results showed that $8.5 \%(n=9)$ of the responded doctors prescribe exercise as a preventative or management method of chronic disease associated to obesity (diabetes, hypertension...). Only
$0.9 \%(n=1)$ of questioned doctors prescribe physical exercise in case of musculoskeletal problem associated to pregnancy. Figure 2 below shows the modality of exercise prescription used by our doctors.

After obtained useful information on Burundi doctors' practices regarding physical exercise prescription, the researcher was interested in knowing whether questioned doctors agree that sedentary way of life during pregnancy can be source of musculoskeletal problem. To this question, all participants to the present study confirmed this association between pregnancies and musculoskeletal problem.

Despite this knowledge, study results showed that all questioned Burundi doctors didn't agree to the importance of physical exercise prescription for the prevention and management of musculoskeletal problems during pregnancy. In fact, $90.4 \%(n=95)$ feel that exercise prescription is not important at all in the prevention

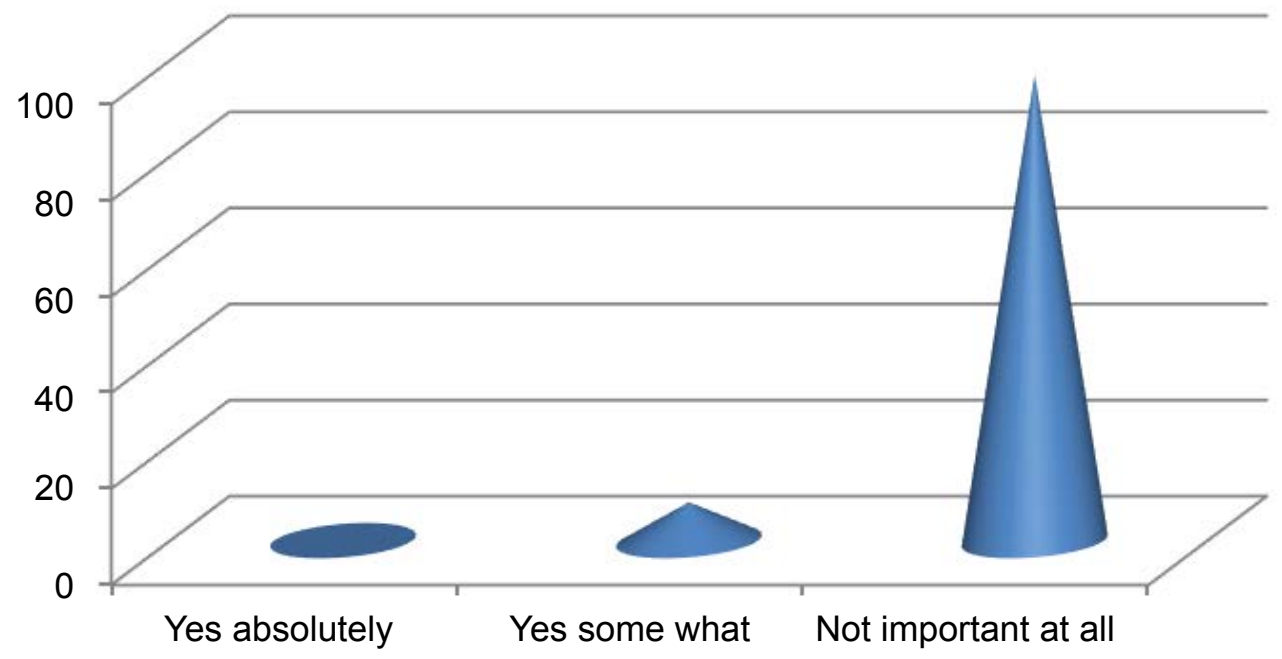

Figure 3: Attached importance on physical exercise in prevention and management of musculoskeletal related to pregnancy.

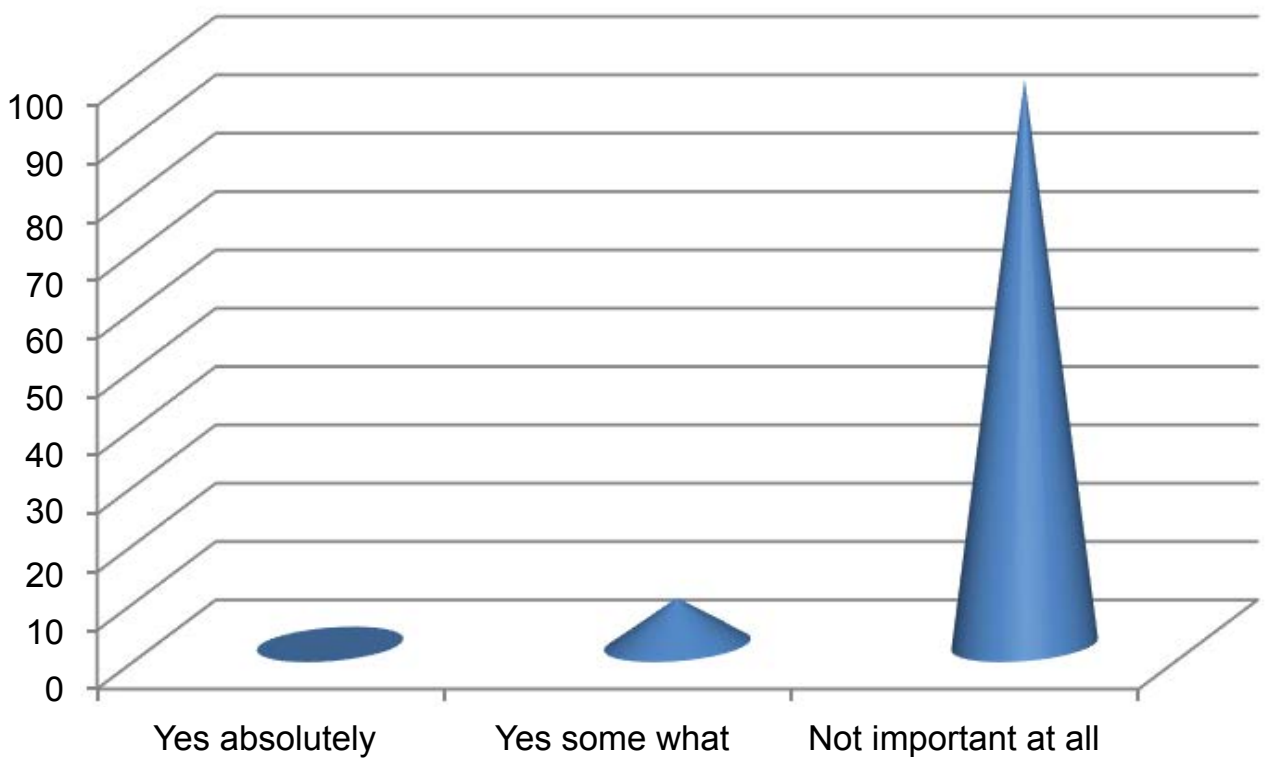

Figure 4: Knowledge regarding international recommendation on physical exercise practice. 
and management of musculoskeletal problems related to pregnancy; $8.5 \%$ of participant to the study hesitate on the importance of physical exercise in prevention and management of musculoskeletal problem associated to pregnancy; while $0.9 \%$ of them feel that physical exercise prescription can be a useful tool to prevent and manage the named problem. Figure 3 shows how important are physical exercise in musculoskeletal problem management for our doctors.

However, the World Health Organization (WHO) recommend urgent preventative action and this action must include an increased emphasis on physical activity as a public health and medical intervention (WHO 2006). And our Burundi doctor's knowledge on international recommendation regarding physical exercise practice is unknown. In this situation, it is important to evaluate our responders on this knowledge. Results have showed that $92.38 \%(n=97)$ do not know any more the international recommendation on physical exercise practice; and $7.6 \%(n=8)$ showed a confused knowledge on this international recommendation. Figure 4 shows our doctor's awareness on international recommendation regarding physical exercise practice.

The present study has assessed possible reasons or barriers which withhold doctors from prescribing exercise to their patients. Some of reasons gathered through the present study are:

$\checkmark \quad$ Lack of adequate skills to provide such prescription or counseling;

$\checkmark \quad$ There is no adequate and standardized form of physical exercise prescription;

$\checkmark \quad$ Doctors support that follow-up support regarding physical exercise practice and its patterns constitute a big problem to exercise prescription;

$\checkmark \quad$ Lack of physicians networking in Burundi where doctors can refer the patient for further training;

$\checkmark$ Doctors feel that physical exercise prescription constitute a promotion of physical exercise rather than medical carrier promotion. And they feel that this is Ministry of youth and sport's Ministry rather than the Ministry of Health.

\section{Discussion}

The discussion of our study results was organized in this order: Doctors' practices regarding physical exercise prescription was firstly discussed, secondly attitudes of doctors regarding physical exercise prescription was discussed and finally doctor's knowledge on international recommendation regarding physical exercise practice.

\section{Doctor's practices regarding physical exercise}

Our study results showed that le clear majority of participants do never prescribe physical exercise to pregnant women. In fact, $90.4 \%$ of participants claimed that they do never prescribe physical exercise in situa- tion of pregnancy. This finding is quite like study results of [17]. In their study on the use of physical exercise as therapy in primary care, they found that physical exercise is taken by doctors as inefficient method, compare to other chemical drugs frequently used in primary care. Also, similar results have been obtained by [18].

In their study on physical exercise prescription, they arrived on results like us. However, in other Physical activity on prescription has been in use since the eighties in for example Sweden and New Zealand. The use of the method has grown in recent years and was applied by all county councils in Sweden in 2008.

For these who reported prescribing physical exercise to their patients, through results, the main way of prescription is "verbal", no one provide written prescription. This study results on the way of exercise prescription is comparable to the study results of [19]. In fact, Marius evaluated the knowledge and attitude of South African Doctors regarding physical exercise prescription. In his study, Doctor Marius found that $68.3 \%$ of participants to his study claimed to provide verbal instruction to their patients, while only $12.8 \%$ of gave a written prescription for the patient. In literature, other researcher reported $3 \%$ of written prescription by doctors [20]. This lack of written exercise prescription among Burundi doctors indicate that there are unaware or ignorant the fact that written prescriptions provided in addition to verbal advice may enhance the effectiveness of interventions [21,22]. In the literature, researchers affirmed that receiving a prescription for exercise from your General Practitioner (GP) represents a well-understood interaction between the patient and the doctor [23] and will constantly remind of the exercise goals set by the general practitioner and the patient [23].

[24] argue that a physical prescription for exercise from a general practitioner is a credible way to communicate changes in lifestyle to patients which focuses on empowerment of the patient to take control of his/her preventative healthcare instead of just removing symptoms by using drugs.

It is also important to note that physical activity is a very important aspect of good health. According to [25] exercise is very important irrespective of age and especially important for pregnant women as it helps them overcome pregnancy related complications and maintain good physical fitness. In addition, exercise during pregnancy not only helps in maintaining musculoskeletal fitness but also helps in controlling weight blood glucose, to cope with various psychological and physical stresses [25]. That why we affirm that this lack of physical exercise prescription observed in Burundi constitutes a handicap to good health promotion.

\section{Attitudes of Burundi doctors regarding physical exercise prescription}

According to responses provided in the present study, 
Burundi doctors working in maternity department showed a negative attitude toward physical exercise prescription to pregnant women. This negative attitude is reflected in our participant's responses. In fact, most doctors participating in the study (90.4\%) feel that physical exercise prescription is not important at all in the management and prevention of musculoskeletal problems associated to pregnancy. Moreover, they don't agree that sedentary can be a source of musculoskeletal problem in pregnant women.

This misunderstanding of the cause of musculoskeletal problem in pregnancy may be the vector of our doctor's negative attitudes to this problem. Our results are opposed to attitudes found in the literature.

In fact, [26] found in his research that $98 \%$ of doctors think that physical exercise promotion should be within a scope of general practitioner practice. [27] also arrived at conclusions like sallis results. This negative attitude of our doctors may be explained by their habit regarding exercising their bodies. The literature shows that the more doctors exercise themselves, the more they will prescribe physical exercise to their patient [28].

Consider Burundi doctors attitudes and practices regarding to exercise prescription in case of pregnancy, the rate of exercise prescription as therapy or tool of prevention is quite low and even equal to zero. The present study has assessed some of barriers to this exercise prescription.

Our responders said that the prescription of physical exercise take the form of: 1) The type of activity (eg, walking), 2) Intensity of activity (eg, light), 3) Duration of activity (eg, 20-30 min), and 4) Frequency of activity (eg, 5 days a week), and they feel that they are not licensed for such prescription.

Moreover, the key is that prescription is based on the individual situation and a patient-centered conversation or dialogue. The patient's current health status, experience, and own desire are important parts of the dialogue between the patient and the health professional. The lack of patient-initiated physical activity discussion constitutes a big obstacle to exercise prescription by doctors. Also, the lack of physical activity prescription protocol in their studies background is another obstacle to physical exercise counseling or prescription. The monitoring of prescribed physical exercise and its follow-up is the biggest constrain to prescribe physical exercise during pregnancy as revered by doctors.

However, despite all these constraints faced by doctors in physical exercise prescription, we remain convinced that they can play a reading role in the promotion of physical activity and as result promoting good health. Population considers health care professionals as credible and trusted interlocutors on good health promotion and education. Advices on increasing physical activity level to combat sedentarily constitute good health promotion.
Physical activity prescription constitutes an integral part of a lot of pathologies prevention including musculoskeletal problems related to pregnancy. And the prevention is the main task assigned to healthcare professionals.

Solid and updated knowledge about the scientific evidence on physical activity and health as well as recent recommendations and guidelines is required among health care professionals to give advice and support to patients who need to increase their physical activity. What about the knowledge of our doctors regarding these guidelines and international recommendations?

\section{Doctors knowledge's regarding physical activity recommendation}

Through our study results, it is evident that Burundi doctors are unaware regarding physical exercise practice and international recommendation. In fact, $46.66 \%$ of participants in the study ignore absolutely these international recommendations, while $26.66 \%$ of participants hesitate on these international recommendations regarding physical activity practices. The literature shows that South African Doctors are more knowledgeable than Burundians on international recommendation regarding physical activity. In fact, Doctors [19] in his study found that $54.6 \%$ of participants knew that an adult should engage in PA at least twice a week. Less than halve $(43.2 \%, n=136)$ knew that the weekly exercise should entail a combination of both moderate and vigorous exercise, while a mere $22.5 \%$ ( $n=71$ ) knew that ideally a person should engage in vigorous exercise three times a week. This weakness in knowledge among Burundi doctors is another handicap to exercise prescription for their patients. However, a good knowledge on this guideline is not enough in itself. The caregiver attitude towards the importance of lifestyle and lifestyle interventions is also essential. These guidelines have been elaborated by the American College of Sport Medicine and the American Heart Association [28].

\section{Conclusion}

$\checkmark$ Burundi doctors are not aware on international recommendation regarding physical exercise practice for good health;

$\checkmark$ Physical exercise prescription is not integrated in everyday primary care particularly in case of pregnancy;

$\checkmark$ Burundi doctors present a negative attitude regarding physical exercise prescription particularly during pregnancy;

$\checkmark$ A pedagogic skill is required in addition to the scientific knowledge in attempt to persuade doctors to include physical exercise prescription in the prevention and management of diver's pathologies including musculoskeletal problems associated to pregnancy.

\section{References}

1. Gordon M (2002) Maternal physiology in pregnancy. In: 
Gabbe S, Niebyl J, Simpson, Obstetrics: Normal and problem pregnancies. ( $4^{\text {th }}$ edn), New York Churchill Livingstone.

2. Rapini $R$ (2004) The skin and pregnancy. In: Creasy $R$, Resnik R, Lams J, Maternal- foetal medicine: Principles and practice. ( $5^{\text {th }}$ edn), Philadelphia: Saunders.

3. Dorothy C, Cynthia F (2014) The impact of pregnancy on foot health. Malta Journal of Health Science.

4. Goldberg J, Besser P, Selby-Silverstein L (2001) Changes in foot function throughout pregnancy. The American College of Obstetricians and Gynecologists 97: 39.

5. Pennick V, Liddle SD (2013) Interventions for preventing and treating pelvic and back pain in pregnancy. Cochrane Database Syst Rev 8: CD001139.

6. Van De Pol G, Van Brummen HJ, Bruinse HW, Heintz AP, Van Der Vaart CH (2007) Pregnancy-related pelvic girdle pain in the Netherlands. Acta Obstet Gynecol Scand 86: 416-422.

7. Mens JM, Vleeming A, Stoeckart R, Stam HJ, Snijders CJ (1996) Understanding peripartum pelvic pain. Implications of a patient survey. Spine (Phila Pa 1976) 21: 1363-1369.

8. Tobias DK, Zhang C, van Dam RM, Bowers K, Hu FB (2011) Physical activity before and during pregnancy and risk of gestational diabetes mellitus: a meta-analysis. Diabetes Care 34: 223-229.

9. Kieffer EC, Willis SK, Arellano N, Guzman R (2002) Perspectives of pregnant and postpartum latino women on diabetes, physical activity, and health. Health Educ Behav 29: 542-556.

10. Sternfeld B (1997) Physical activity and pregnancy outcome. Review and recommendations. Sports Med 23: 33-47.

11. Melzer K, Schutz Y, Soehnchen N, Othenin-Girard V, Martinez de Tejada B, et al. (2010) Effects of recommended levels of physical activity on pregnancy outcomes. Am J Obstet Gynecol 202: 266.

12. World Health Organization (2006) Chronic diseases and health promotion. Switzerland: WHO Press.

13. Blair SN (2009) Physical inactivity: the biggest public health problem of the 21st century. Br J Sports Med 43: 1-2.

14. Elley CR, Kerse N, Arroll B, Robinson E (2003) Effectiveness of counselling patients on physical activity in general practice: cluster randomised controlled trial. BMJ 326: 793.

15. Hellénius ML (2011) Prescribing exercise in clinical practice. Current Cardiovascular Risk Reports 5: 331-339.

16. Dauenhauer J, Podgorski C, Karuza J (2006) Prescribing exercise for older adults: A needs assessment comparing primary care physicians, nurse practitioners, and physician assistants. Gerontology \& geriatrics Education. Gerontol Geriatr Educ 26: 81-100.
17. Calderón C, Balagué L, Cortada JM, Sánchez A (2011) Health promotion in primary care: how should we intervene? A qualitative study involving both physicians and patients. BMC Health Serv Res 11: 62.

18. Person G, Brorsson A, Hansson E, Throcin N, Strandberg EL (2013) Physical activity prescription from general practitionner's perspective -a qualitative study. BMC Fam Pract 14: 128 .

19. Marius GR, Schoeman M, Holtzhausen LJ (2014) Exercise prescription; Knowledge, practice and attitudes among South African Doctors.

20. Sørensen JB, Kragstrup J, Kjaer K, Puggaard L (2007) Exercise on prescription: trial protocol and evaluation of outcomes. BMC Health Serv Res 7: 36.

21. Marcus $B H$, Williams DM, Dubbert PM, Sallis JF, King AC, et al. (2006) Physical activity intervention studies: what we know and what we need to know: a scientific statement from the American Heart association council on nutrition, physical activity, and metabolism (Subcommittee on physical activity); council on cardiovascular disease in the young; and the interdisciplinary working group on quality of care and outcomes. Circulation 114: 2739-2752.

22. Smith BJ, Bauman A, Bull F, Booth M, Harris M (2000) Promoting physical activity in general practice: a controlled trial of written advice and information materials. $\mathrm{Br} \mathrm{J}$ Sports Med 34: 262-267.

23. Swinburn BA, Walter LG, Arroll B, Tilyard MW, Russell DG (1998) The green prescription study: a randomized controlled trial of written exercise advice provided by general practitioners. Am J Public Health 88: 288-291.

24. Huber M, Knottnerus JA, Green L, van der Horst $H$, Jadad AR, et al. (2011) How should we define health? BMJ 343: 4163.

25. Rachana N, Simeen V, Chetna G, Vijaya K, Amitesh N, et al. (2015) Awareness of antenatal exercise among pregnant woman in tertial care center, Mangalore, India. IJAMSCR 3: 243-247.

26. Sallis RE (2009) Exercise is medicine and physicians need to prescribe it! Br J Sports Med 43: 3-4.

27. Matteson KA, Anderson BL, Pinto SB, Lopes V, Schulkin $J$, et al. (2011) Surveying ourselves: examining the use of aweb-based approach for a physician survey. Evaluation \& the Health Professions 34: 448-463.

28. Haskell WL, Lee IM, Pate RR, Powell KE, Blair SN, et al. (2007) Physical activity and public health: Updated recommendation for adults from the American College of Sports Medicine and American Heart Association. Med Sci Sports Exerc 39: 1423-1434. 